\section{RMD Open}

Rheumatic \&

Musculoskeletal Diseases

\title{
Imaging of diffuse idiopathic skeletal hyperostosis (DISH)
}

Reuven Mader (D) , ${ }^{1}$ Xenofon Baraliakos (D) , ${ }^{2}$ Iris Eshed, ${ }^{3}$ Irina Novofastovski, ${ }^{1}$ Amir Bieber, ${ }^{1}$ Jorrit-Jan Verlaan, ${ }^{4}$ David Kiefer (D) ,,6 Nicola Pappone, ${ }^{7}$ Fabiola Atzeni ${ }^{8}$

\section{ABSTRACT}

Diffuse idiopathic skeletal hyperostosis (DISH) is a condition characterised by calcification and ossification of ligaments and entheses. The condition usually affects the axial skeleton, in particular, at the thoracic segment, though also other portions of the spine are often involved. DISH often involves also peripheral tendinous and/or entheseal sites either alone, or in association with the involvement of peripheral joints. At times, new bone formation involves the bone itself, but sometimes it involves joints not usually affected by osteoarthritis (OA) which result in bony enlargement of the epiphysis, joints space narrowing and a reduced range of motion. Because of the entheseal involvement, DISH can be mistaken for seronegative spondyloarthropathies or for a "simple" $O A$. Furthermore, other implications for the recognition of DISH include spinal fractures, difficult intubation and upper endoscopies, decreased response rates in DISH with concomitant spondyloarthritides, and increased likelihood to be affected by metabolic syndrome and cardiovascular diseases. This Atlas is intended to show the imaging finding in DISH in patients diagnosed with the condition by the Resnick classification criteria.

\section{Afula, Israe}

${ }^{2}$ Rheumatologie Ruhr-Universität Bochum, Rheumazentrum Ruhrgebiet, Herne, Germany ${ }^{3}$ Department of Radiology, Musculoskeletal imaging Center, Tel Aviv University Israel, Tel Aviv, Israel

${ }^{4}$ Department of Orthopedics, University Medical Center Utrecht, Utrecht, The Netherlands

${ }^{5}$ Rheumatologie, Rheumazentrum Ruhrgebiet, Herne, NRW, Germany

${ }^{6}$ Rheumatologie, RuhrUniversitat Bochum, Bochum, Germany

${ }^{7}$ Rheumatology Rehabilitation, Fondazione S. Maugeri, Telese Terme, Italy

${ }^{8}$ Rheumatology Unit, Department of Internal and Experimental Medicine, University of Messina, Messina, Italy

Correspondence to Dr Xenofon Baraliakos; baraliakos@me.com

\section{INTRODUCTION}

Diffuse idiopathic skeletal hyperostosis (DISH), is a condition characterised by calcification and ossification of ligaments and entheses. The condition usually affects the axial skeleton, in particular at the thoracic segment though also other portions of the spine are often involved. DISH often involves also peripheral tendinous and/or entheseal sites either alone, or in association with peripheral joints' involvement. ${ }^{1}$ At times, new bone formation involves the bone itself, but sometimes it involves joints not usually affected by osteoarthritis (OA), which result in bony enlargement of the epiphysis, joints space narrowing and a reduced range of motion. ${ }^{2}$ Because of the entheseal involvement, DISH can be mistaken for seronegative spondyloarthropathies or for a 'simple' OA. ${ }^{3}$ Furthermore, other implications for the recognition of DISH include spinal fractures, difficult

\section{Key messages}

What is already known about this subject?

- DISH is a relatively common musculoskeletal condition appearing in the rheumatology practice

- Although the clinical symptoms are common to other musculoskeletal diseases, the diagnosis is mainly being made using images of the axial and the peripheral skeleton

What does this study add?

- We here present a comprehensive imaging Atlas of various, common and rare manifestations of DISH

How might this impact on clinical practice?

- This Atlas may help clinicians in daily practice as reference of unclear images of patients who present with clinical symptoms suggestive of DISH and where imaging may be inconclusive.

intubation and upper endoscopies, decreased response rates in DISH with concomitant spondyloarthritides, and increased likelihood to be affected by metabolic syndrome and cardiovascular diseases. This atlas is intended to show the imaging finding in DISH in patients diagnosed with the condition by the Resnick classification criteria. ${ }^{1}$

\section{THORACIC SPINE}

In the initial stages of the disease, small bony areas in front of the disk space can be

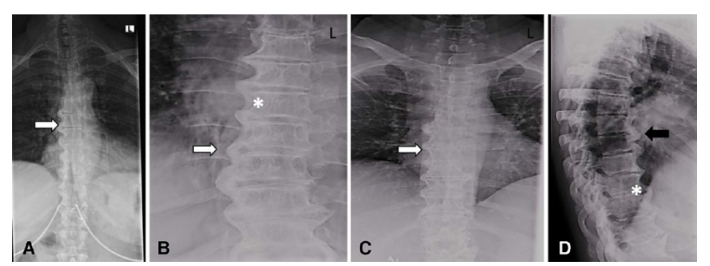

Figure 1 (A-C) Posterior-anterior and (D) lateral: large right-sided flowing bridges (white arrows). Note the space between the ligament and the vertebral body $\left({ }^{*}\right)$. Thick flowing ossification of the anterior lateral ligament is shown (black arrow). 

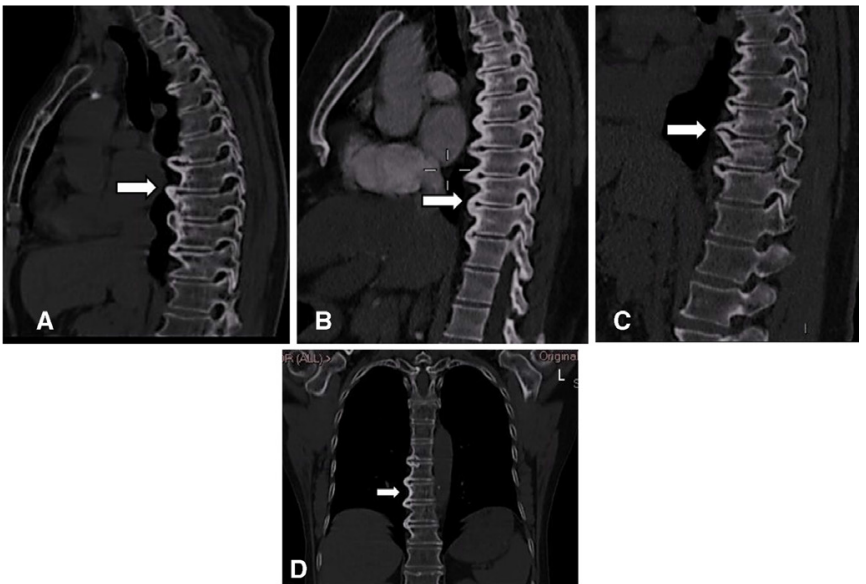

Figure 2 (A-C) Sagittal: CT scan images of anterior flowing osteophytes (arrows). (D) Coronal: dish of the thoracic spine (arrow) reconstructed from the chest CT scan. $\mathrm{L}=$ left.

observed in the sagittal projection. Then, laminar ossifications appear in the medial-inferior and medial-superior portion of the anterior and lateral vertebral margins. In the following stages, enthesophytes do elongate and thicken, especially on the anterior and right sides of the vertebral bodies. Between the newly formed bone and the anterior border of the vertebral body, a 'linear radiolucency' may be detected. Involvement of the T-spine is probably the most characteristic radiographical finding. Ankylosis is often incomplete. ${ }^{4}$

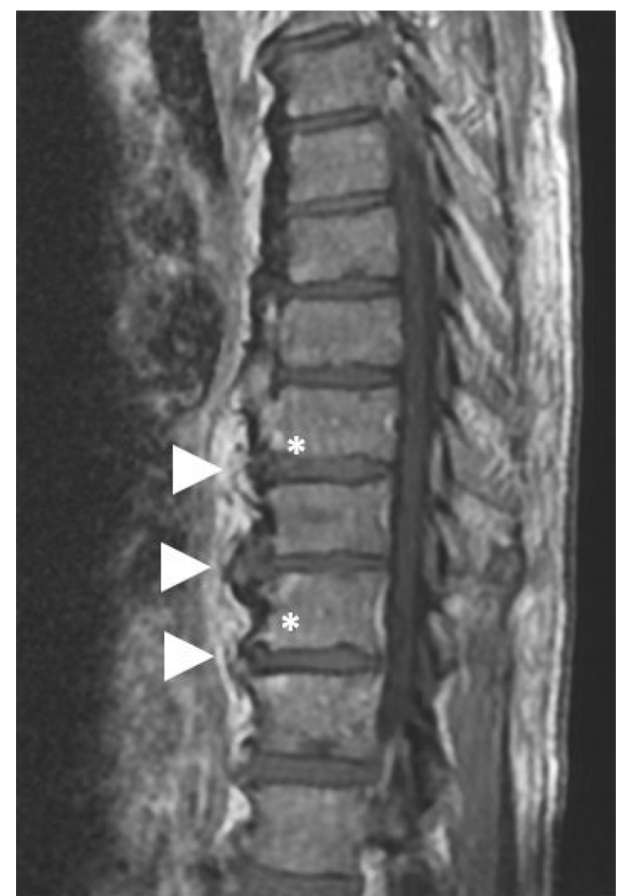

Figure 3 Sagittal T1-weighted image of the thoracic spine. Characteristic flowing osteophytes between four thoracic vertebrae (arrowheads) accompanied by small fatty corners in the anterior endplate of the corresponding vertebrae $\left({ }^{*}\right)$.
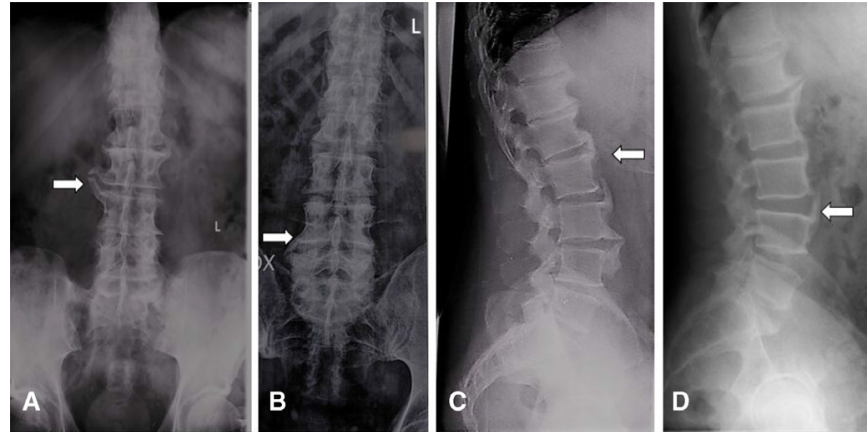

Figure 4 (A) Posterior-anterior: L3-L4 right-sided large bridging osteophyte (arrow). (B) Posterior-anterior: ossification of the anterior lateral ligament with nonmarginal osteophytes (arrow). (C) Lateral: large non-marginal osteophytes. Note the vertical spur of L3 parallel to $L 2$ vertebral body (arrow). (D) Lateral: large non-marginal osteophytes. Note the vertical spur of L4 with normal C3-C4 intervertebral space (arrow). L = left

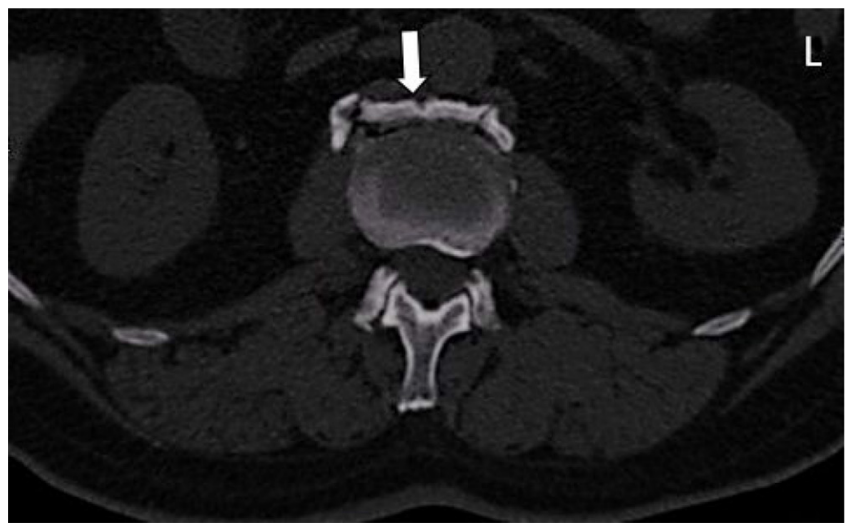

Figure 5 Transversal: CT scan image showing ossification of the anterior lateral ligament separated from the vertebral body (arrow). $L=$ left.

\section{LUMBAR SPINE}

The upper lumbar segments are involved in a large percentage of cases. Radiographical abnormalities along the anterior aspect of the lumbar spine are similar to those of the cervical spine. Unlike the thoracic spine, the flowing ossifications are equally frequent on the right and left sides of the lumbar spine. One can observe ossifications of the spinous processes and of the interspinous ligaments. The narrowing of the intervertebral space is generally classified as mild to moderate. Degenerative changes in apophyseal joints can occur in the lower lumbar spine and in the lumbosacral junction too. Due to the hyperostosis, spinal stenosis is not rare. ${ }^{5}$

\section{CERVICAL SPINE}

The hyperostotic process develops along the lower half of the anterior border of the vertebral body: morphological aspects are described appears as 'falling drop' or as prevertebral drop at the front of the vertebrae. Subsequently, these kind of 'drops' tend to grow, forming spurs (enthesophytes) until they band together; then, the spurs 


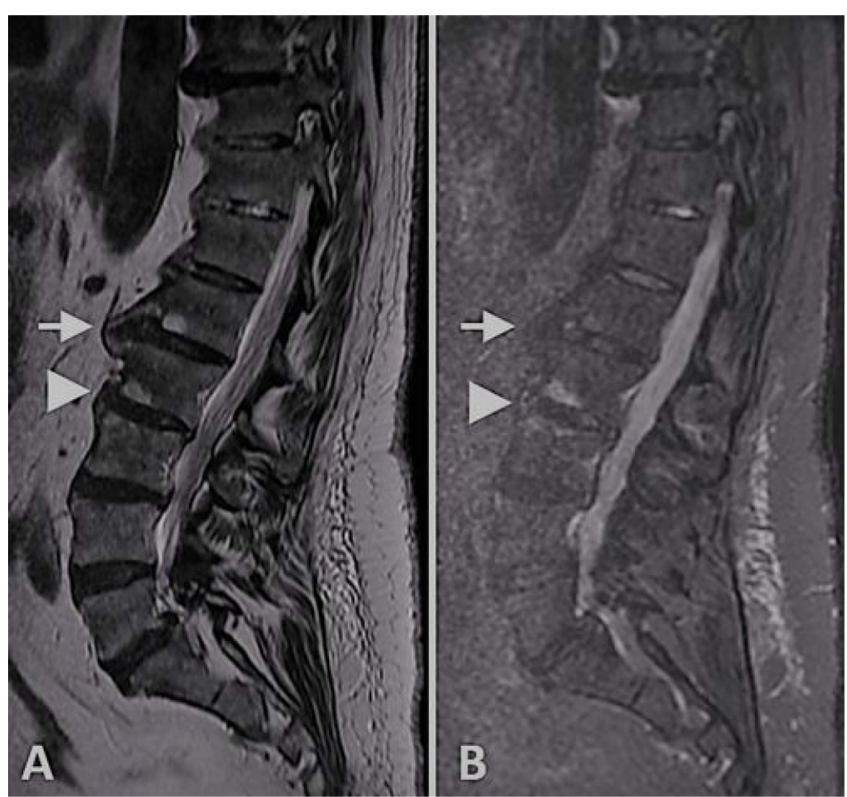

Figure 6 Sagittal T2-weighted (A) and Short tau inversion recover (STIR) (B) images of the lumbar spine of a subject with diffuse idiopathic skeletal hyperostosis. Characteristic bony bridges connecting the anterior part of L1-L2 (arrows) and L2-L3 (arrowheads) can be seen.

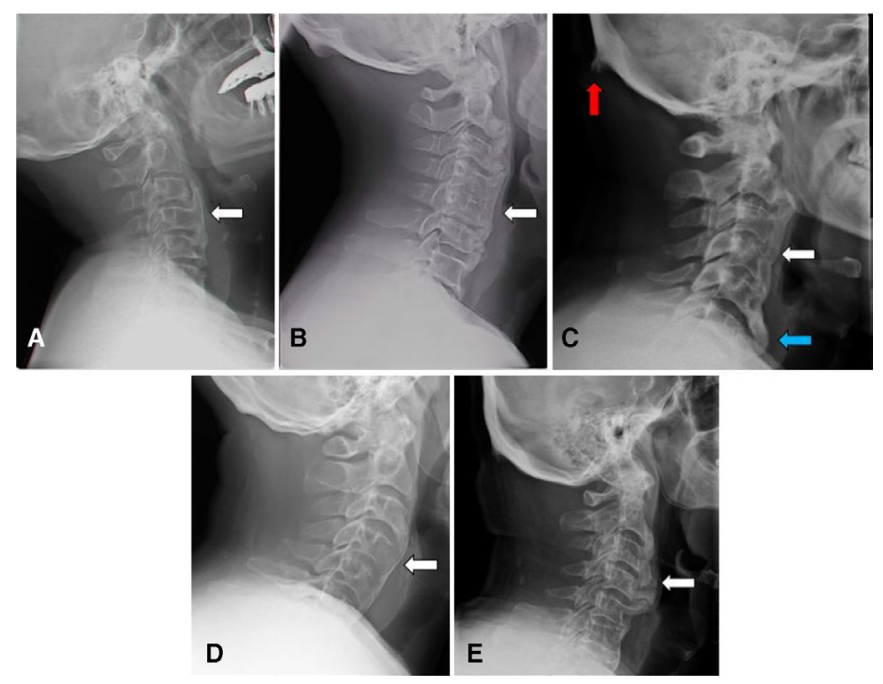

Figure 7 (A) Anterior osteophyte independent from C4 (arrow). (B) Thickening and ossification of anterior longitudinal ligament (arrow). (C) Thickening and ossification of anterior longitudinal ligament (arrow), large enthesophytes (blue arrow) and nuchal enthesopathy (red arrow). (D) Flowing thickened anterior lateral ligament (arrow). (E) Patient with diffuse idiopathic skeletal hyperostosis and swallowing difficulties (arrow).

expand on the superior and anterior tracts of the underlying vertebral body, leading in turn to the formation of 'candle flame', 'parrot-beak' image or 'bridge'. The cervico-occipital junction may be involved with the vertebral bodies showing increased diameters due to bone neoapposition along the margins. At times, the hyperostotic process may involve the odontoid process and the adjacent ligaments. ${ }^{1}$
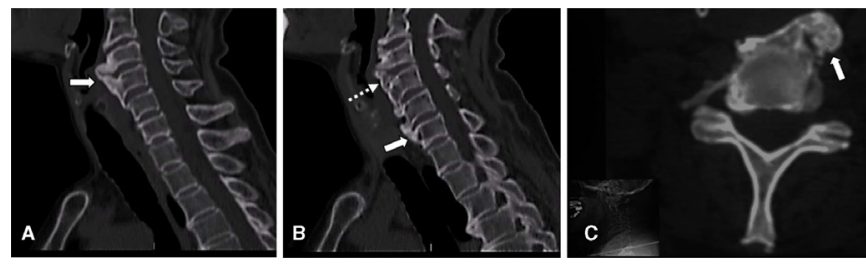

Figure 8 (A) Sagittal: CT large C3-C4 anterior osteophyte (arrow). (B) Sagittal: CT large anterior osteophyte. Note the ossification originating from the discal annulus fibrosus (dotted arrow) and the space between the anterior longitudinal ligament and the vertebral body (arrow). (C)

Transversal: large anterolateral osteophyte (arrow).

\section{PERIPHERAL INVOLVEMENT}

Peripheral involvement in DISH is characterised by several distinctive features:

Involvement of joints usually unaffected by primary OA, such as the elbows, metacarpophalangeal joints, shoulders and others.
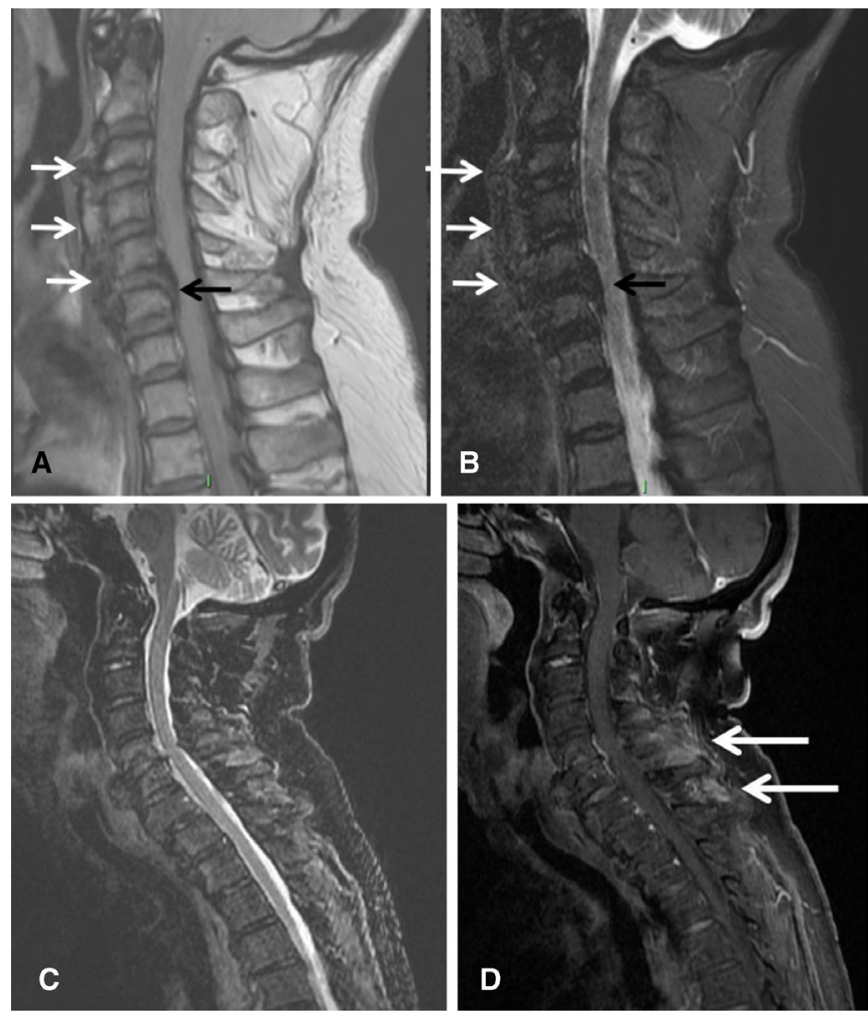

Figure 9 (A) Sagittal T2-weighted and (B) Short tau inversion recover (STIR) images of the cervical spine of a subject with diffuse idiopathic skeletal hyperostosis. Characteristic thick confluent bony bridges connecting the anterior part of vertebrae C3-C6 (white arrows) can be seen. Thick ossification of the posterior longitudinal ligament with low signal intensity in both sequences is seen in the posterior surface of C5-C6 intervertebral space (black arrow). (C) STIR and (D) T1-weigthed with fat suppression after intravenous gadolinium injection. Enthesitis of the posterior interspinus ligament connecting the posterior vertebral processes of C4$\mathrm{C} 7$ can be seen as a high signal intensity after gadolinium injection in (D) (arrows). 


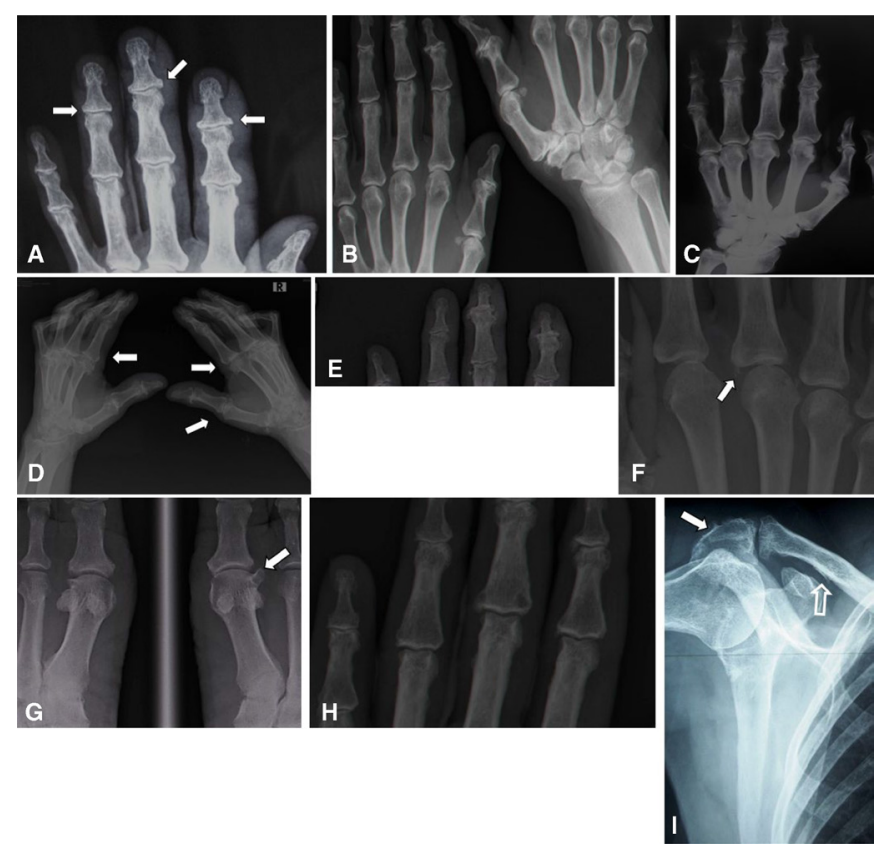

Figure 10 (A) Left hand: enlargement of the base of the distal phalanx (arrows). (B) Hands, both sides: joint space narrowing and exuberant new bone formation of both thumbs' interphalangeal joint and the second left distal interphalangeal joint. New bone formation at the radial margin of the right trapezium. (C) Hypertrophic osteoarticular changes in the interphalangeal joints. Joint space narrowing of the second and third metacarpophalangeal joints with enlargements and osteophytes of the third metacarpal head. (D) Joint space narrowing with exuberant new bone formation (arrows). (E) Hypertrophic/hyperostotic Heberden's nodes. (F) Third metacarpophalangeal capsular ossification (arrow). (G) Large osteophyte of the first metatarsal head (arrow). (H) Capsular ossification of the second PIP, second distal interphalangeal joint and third PIP. (I) Exostosis of the acromion (full arrow). Remodelling of the mid/proximal clavicle (empty arrow). PIP, proximal interphalangeal joint.

Increased hypertrophic changes compared with primary OA. Prominent enthesopathies at various sites adjacent to peripheral joints, including entheseal insertions of joints' capsules, and tendons attachments. Calcification and ossification of entheses in sites other than joints such as the tibial tuberosity, olecranon, Achilles insertion and plantar fascia. ${ }^{2}$

\section{DISH AND ANKYLOSING SPONDYLITIS (AS)}

DISH and AS are the prototypes of hyperostotic diseases of the axial skeleton. ${ }^{6}$ The bone proliferation leads in the long term to ankylosis of the spine and functional impairment. Similarly, due to the local osteoporosis and decreased flexibility of the affected bones, vertebral fractures are frequent in both DISH and AS even after minor impact traumata. ${ }^{7}$ Beyond clinical and genetic differences, the phenotype of new bone formation is different between the diseases. In DISH, the so-called 'chunky bridging osteophytes' which grow as an additional layer on the anterior longitudinal ligament are typical, while

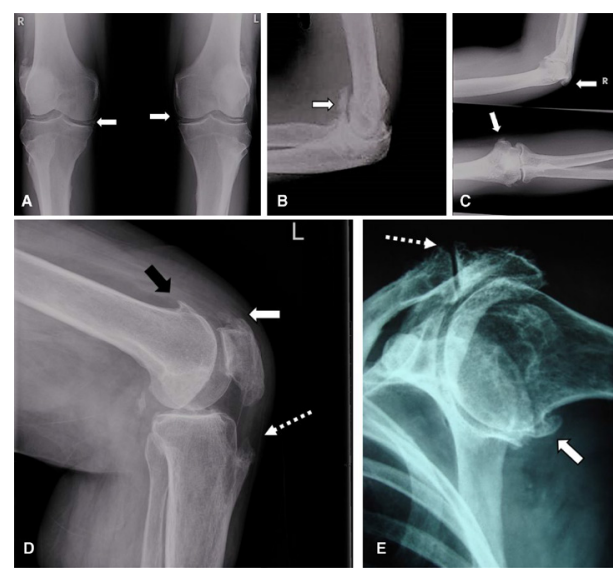

Figure 11 (A) Chondrocalcinosis in a patient with diffuse idiopathic skeletal hyperostosis (arrows). (B) Elbow joint space narrowing with a gigantic osteophyte (arrow). (C) Elbow joint space narrowing with capsular ossification (arrows). (D) Knee: femoral condyle osteophyte (white arrow), ossification of the patellar tendon (black arrow) and large enthesopathy of the tibial tuberosity (dotted arrow). (E) Shoulder: large osteophyte of the humeral head (white arrow) and acromioclavicular degenerative osteophytes (dotted arrow). $L=$ left, $R=$ right.

in AS, the frequently thinner, marginal and symmetrical syndesmophytes which represent an ossification of enthesitic lesions at the area of the attachment of the anterior longitudinal ligament and the annulus fibrosus are more common. ${ }^{6}$ Although initially DISH and AS were

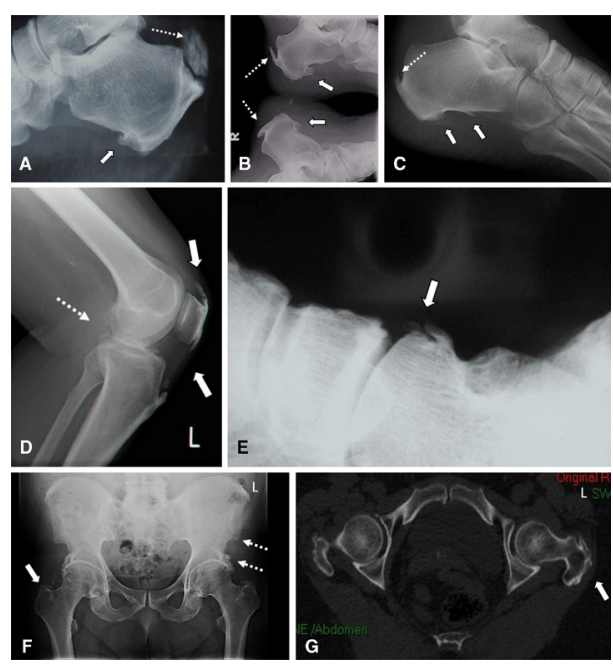

Figure 12 (A) Plantar enthesophyte (arrow) and ossification of the terminal portion of the Achilles tendon (dotted arrow).

(B) Large, bilateral Achilles (dotted arrows) and plantar enthesopathies (arrows). (C) Achilles enthesopathy (dotted arrow). Calcaneal enthesophyte extending along the plantar fascia (arrows). (D) Enthesopathy of the patellar ligament anterior to the patella and at the tibial tuberosity (arrows). Calcification of the posterior knee capsule (dotted arrow). (E) Talonavicular enthesopathy (arrow). (F) Ossification of the hips' joints capsules (dotted arrows). Enthesopathy of the right greater trochanter and the iliac bone margins (arrow). (G) CT: enthesopathy of the left greater trochanter (arrow). L $=$ left, $\mathrm{R}=$ right. 

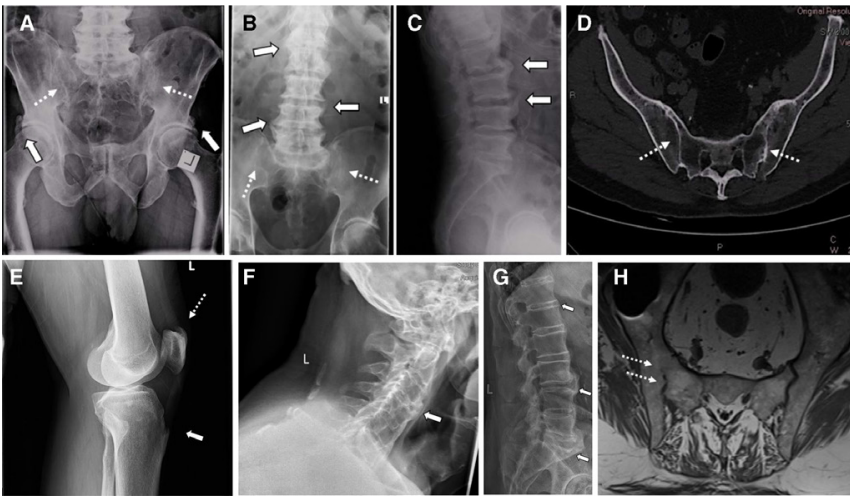

Figure 13 (A) X-ray of the pelvis: hips with coarse capsular ossification (arrows) with grade 4 sacroiliitis (dotted arrows). (B) X-ray of the spine with Sacroiliac joints: coarse asymmetrical osteophytes (arrows) with grade 4 sacroiliitis (dotted arrows). (C) X-ray of the lumbar spine, sagittal: anterior thick bridges and ossification of the anterior lateral ligament. (D) CT SIG: erosive sacroiliitis with anterior osseous bridges (dotted arrows). (E) X-ray of the knee: patellar (dotted arrow) and tibial tuberosity enthesopathies (arrow). X-ray of the cervical (F) and lumbar spine (G) with thickening and ossification of anterior longitudinal ligament (arrows) as well as MRI sacroiliac joint (T1 sequence) with partial ankylosis $(\mathrm{H}$, dotted arrows) in a patient with both diseases diffuse idiopathic skeletal hyperostosis and ankylosing spondylitis. $\mathrm{L}=$ left.

thought to be distinct conditions, recent studies have also reported the simultaneous presence of both diagnoses in some patients. ${ }^{8}$ Structural changes of the spine and the SIJ may occur in DISH and AS. Therefore, MRI sequences may be helpful differentiating the two diseases as, in addition to structural damage, MRI may show inflammatory changes with the typical pattern of AS. In addition, especially in younger patients, radiation exposure also needs to be considered, making MRI a useful tool in this diagnosis.

\section{PELVIS IN DISH}

The pelvis is one of the main extraspinal involved sites in DISH and was therefore suggested to be included in future classification criteria for DISH.

Pelvic enthesopathies are considered highly characteristic of DISH and have been shown to be significantly
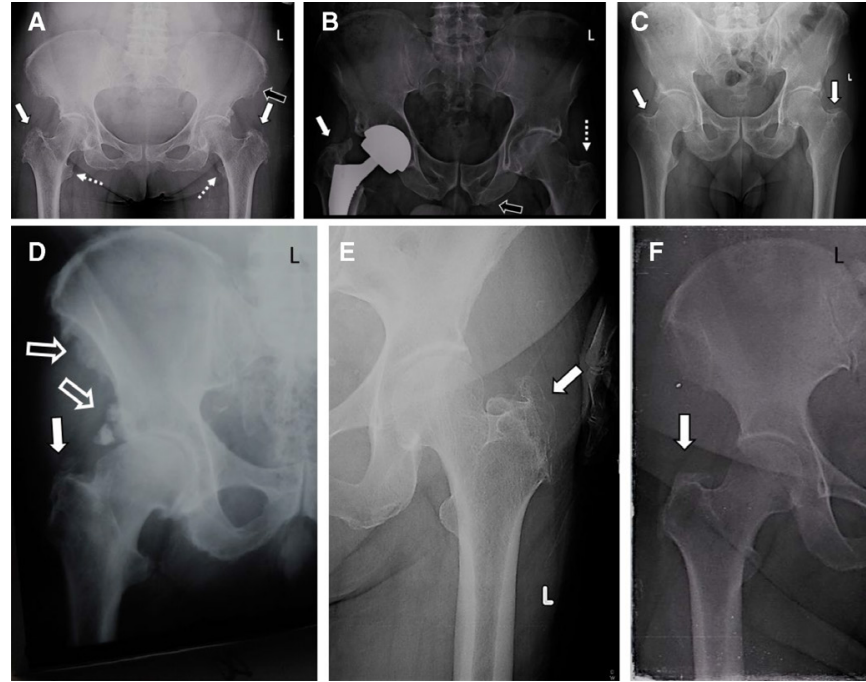

Figure 14 (A) Enthesopathies of the greater trochanter (full arrows), lesser trochanter (dotted arrows), left hip capsule and iliac bone (empty arrow). (B) Heterotropic ossification following right total hip replacement (full arrow) of the left trochanter (dotted arrow) and ischium enthesophytes (empty arrow). (C) Bilateral hip joints' capsule ossification (full arrows). (D) Enthesopathy of the greater trochanter and hip joint capsule (full arrow). Periostitis of the iliac and ischial bones (empty arrows). (E,F) Exuberant trochanteric enthesopathies (arrows). L = left.

more prevalent and more prominent in DISH subjects compared with controls. ${ }^{9}$ Indeed, pelvic insertional tendon enthesopathy on pelvic radiographs and on CT, seen as an ossification or calcification of the entheses, has been found to be a good indicator of the presence of radiographical spinal DISH. ${ }^{10}$ Among the main involved pelvic entheses are the origin of the gluteus medius along the iliac crest, the origin of the adductors on the symphysis pubis, the hamstrings origin on the ischial tuberosity and insertion of the gluteus medius on the greater trochanter.

The sacroiliac joints (SIJs) are of main interest in DISH. On one hand, the Resnick and Niwayama criteria exclude their involvement in order to differentiate DISH from $\mathrm{AS},{ }^{12}$ but on the other hand, recent studies have shown that enthesopathy is also highly abundant in the SIJs of DISH subjects. ${ }^{13}$ Indeed, erosions are seldom seen
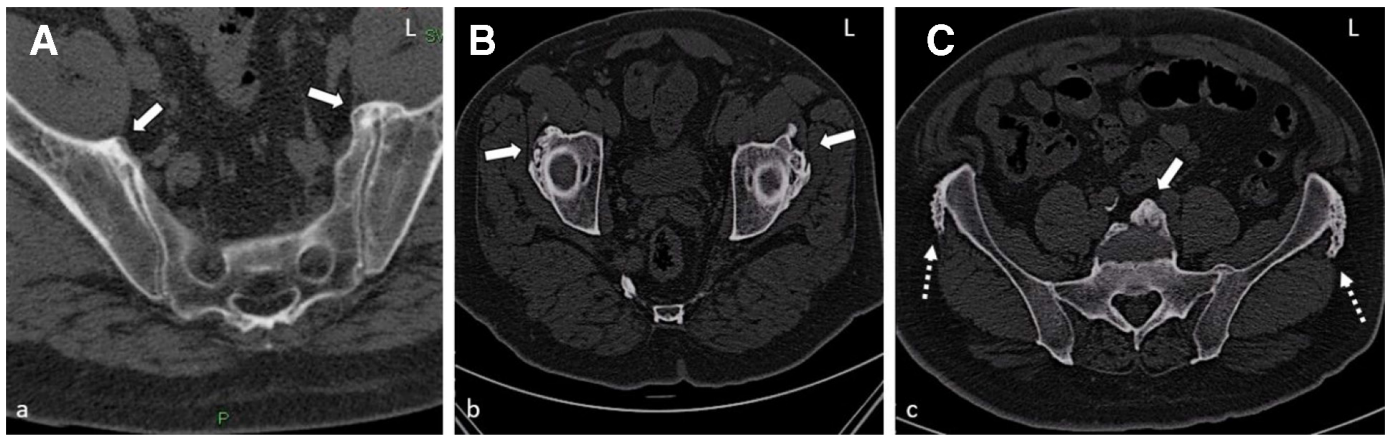

Figure 15 (A) Anterior bridges with normal sacroiliac joints (arrows). (B) Enthesopathy of the great trochanters (arrows). (C) Ossification of anterior lateral ligament (full arrow) and large ileum enthesopathy (dotted arrows). 


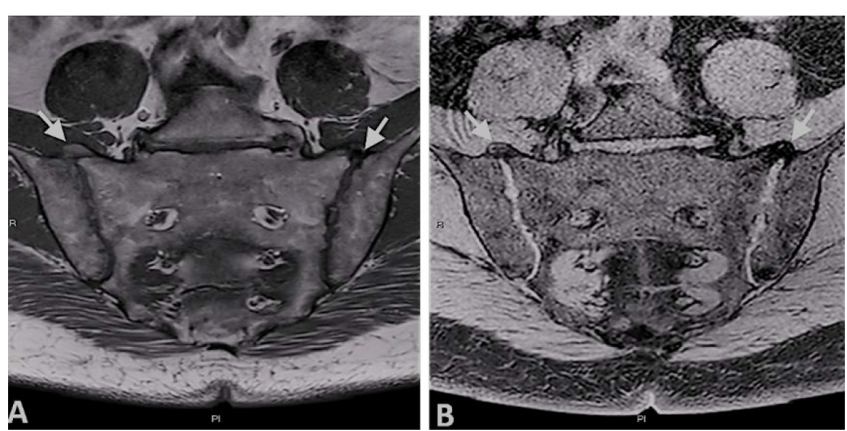

Figure 16 Semicoronal T1-weighted $(A)$ and semicoronal T2-weighted (B) MRI images of the SIJs of a subject with diffuse idiopathic skeletal hyperostosis demonstrating characteristic bilateral bony bridges across the extraarticular, anterior part of the SIJ (arrows). SIJ, sacroiliac joint.

in DISH and indeed differentiate it from AS. ${ }^{11}$ However, enthesopathy of the anterior and posterior ligaments and capsule of the SIJs, as well as bridging, fusing enthesophytes within the joints mimicking joint ankylosis of AS, have been shown to be characteristic of DISH. ${ }^{6}$ Some preliminary MRI studies have described bone marrow oedema in the SIJs of DISH subjects; however, this observation needs further in-depth evaluation and characterisation. ${ }^{12}$

\section{DISH AND SPINAL FRACTURES}

The osseous bridges connecting vertebral bodies ventrally in DISH may cause stiffening of the spinal column, thereby changing biomechanical characteristics. Although appearing robust with the abundantly formed bone, the spinal column in individuals with DISH is actually more prone to fractures compared with non-affected individuals. Fractures may occur after only minor or trivial trauma, are often severely displaced and are frequently accompanied by neurological deficits due to associated spinal cord injury. ${ }^{13}{ }^{14}$ Identifying spinal fractures in individuals with DISH can be challenging due to the radiological presence of degenerative changes, occult fracture lines (although these lines typically run all the way front to back through the spinal segments), and patients not always being able to distinguish between pre-existent and new pain in the back and/or neck. With plain radiographs, spinal fractures in DISH can be easily missed; therefore, a CT scan of the entire spinal column is recommended if a fracture is suspected. MRI scans are helpful in case of suspicion of occult fractures that are difficult to detect with CT. ${ }^{15}$ Hyperextension is the predominant fracture mechanism in DISH (Arbeitsgemeinschaft für Osteosynthesefragen (AO) type B3), a configuration which generally requires long-segment surgical fixation to provide sufficient mechanical stability to allow for fracture healing and prevent (further) neurological deficits. Patients not fit for surgery may be treated conservatively with collar or brace, but some evidence exists showing the complication rates to be higher in non-operatively treated patients when compared with patients undergoing prompt surgical fixation. ${ }^{16}$

\section{THORACIC SPINE}

X-ray images of the thoracic spine are shown in figure 1 . CT images of the thoracic spine are shown in figure 2.

A magnetic resonance image of the thoracic spine is shown in figure 3 .

\section{LUMBAR SPINE}

X-ray images of the lumbar spine are shown in figure 4.

A CT image of the thoracic spine is shown in figure 5.

Magnetic resonance images of the lumbar spine are shown in figure 6 .

\section{CERVICAL SPINE}

X-ray images of the cervical spine are shown in in figure 7. CT images of the cervical spine are shown in figure 8 .

Magnetic resonance images of the thoracic spine are shown in figure 9 .

\section{Peripheral joint involvement}

X-ray images of small joints are shown in figure 10.
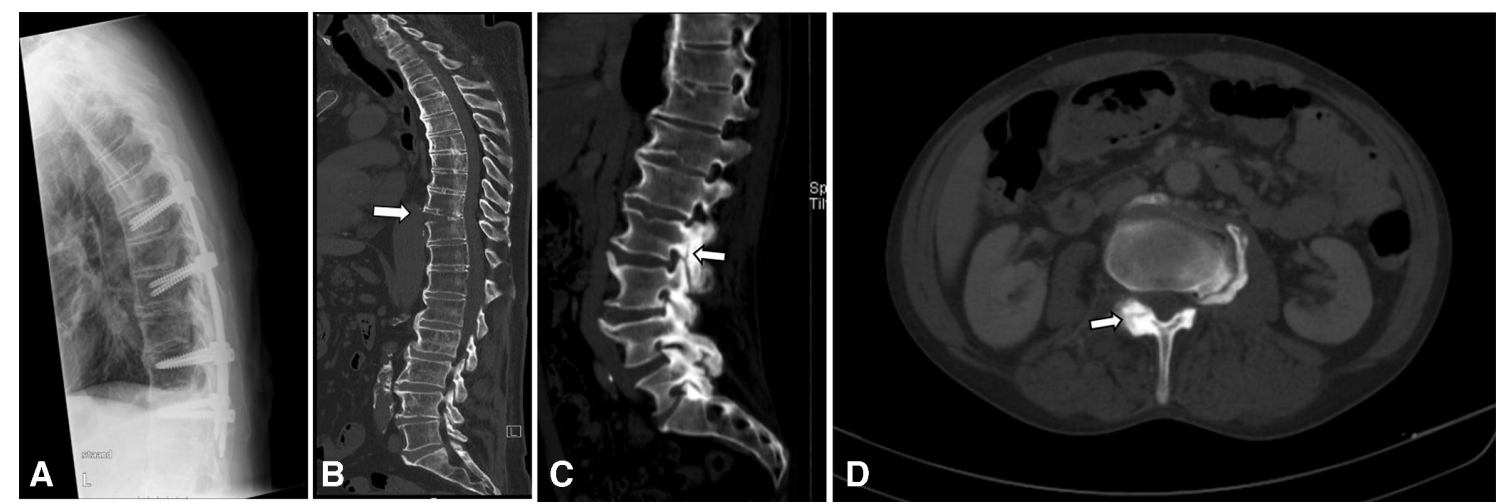

Figure 17 (A) X-ray: percutaneous fixation Th7-Th12. (B) CT scan: hyperextension fracture of Th10 (arrow). (C) CT scan (sagittal) of a patient with fracture of the pedicle of the lumbar vertebrae 2 (arrow). (D) CT scan (transversal) of a patient with fracture of the pedicle of the lumbar vertebrae 2. 


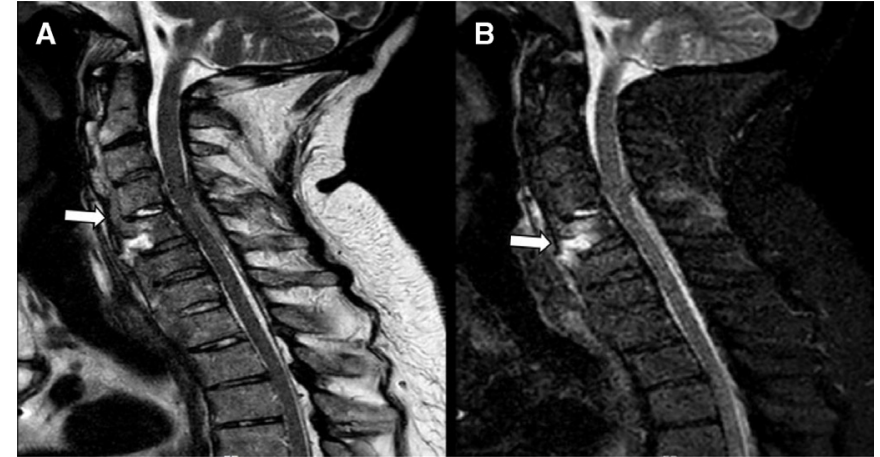

Figure $18(A, B)$ Vertebral fracture (arrows) of C5 in the T1weighted $(A)$ and Short tau inversion recover (STIR) sequence (B).

\section{Peripheral joint involvement}

$\mathrm{X}$-ray images of large joints are shown in figure 11.

\section{Enthesopathies in DISH}

X-ray and CT images of enthesopathies are shown in figure 12.

\section{DISH and AS}

DISH and AS in X-ray and CT are shown in figure 13.

\section{Changes in the pelvis in DISH}

X-ray changes in the pelvis in DISH are shown in figure 14.

CT changes in the pelvis in DISH are shown in figure 15.

MRI changes in the pelvis in DISH are shown in figure 16.

\section{Fractures in DISH}

Fractures in DISH in X-ray and CT are shown in figure 17.

Fractures in DISH in MRI are shown in figure 18.

Contributors All authors collected the images, agreed on the content, and revised and approved the manuscript. All Authors contributed with images and/or comments to the entire imaging collection of this Atlas.

Funding The authors have not declared a specific grant for this research from any funding agency in the public, commercial or not-for-profit sectors.

Competing interests None declared.

Patient consent for publication Not required.

Provenance and peer review Not commissioned; externally peer reviewed.

Data availability statement All data relevant to the study are included in the article or uploaded as supplementary information. There are no data to serve as a background of this article.

Open access This is an open access article distributed in accordance with the Creative Commons Attribution Non Commercial (CC BY-NC 4.0) license, which permits others to distribute, remix, adapt, build upon this work non-commercially, and license their derivative works on different terms, provided the original work is properly cited, appropriate credit is given, any changes made indicated, and the use is non-commercial. See: http://creativecommons.org/licenses/by-nc/4.0/.

\section{ORCID iDs}

Reuven Mader http://orcid.org/0000-0001-7904-8506

Xenofon Baraliakos http://orcid.org/0000-0002-9475-9362

David Kiefer http://orcid.org/0000-0003-1602-7649

\section{REFERENCES}

1 Resnick D, Niwayama G. Radiographic and pathologic features of spinal involvement in diffuse idiopathic skeletal hyperostosis (DISH). Radiology 1976;119:559-68.

2 Mader R, Sarzi-Puttini P, Atzeni F, et al. Extraspinal manifestations of diffuse idiopathic skeletal hyperostosis. Rheumatology 2009:48:1478-81.

3 Olivieri I, D'Angelo S, Palazzi C, et al. Diffuse idiopathic skeletal hyperostosis: differentiation from ankylosing spondylitis. Curr Rheumatol Rep 2009;11:321-8.

4 Resnick DNG. Diagnosis of bone and joint disorders. Philadelphia: WB Saunders, 1988: 1563-615.

5 Ott V. [Senile ankylosing hyperostosis of the vertebral column (Forestier-Rotès)]. Z Rheumaforsch 1952;11:95-105.

6 Baraliakos X, Listing J, Buschmann J, et al. A comparison of new bone formation in patients with ankylosing spondylitis and patients with diffuse idiopathic skeletal hyperostosis: a retrospective cohort study over six years. Arthritis Rheum 2012;64:1127-33.

7 Mader R, Verlaan J-J, Buskila D. Diffuse idiopathic skeletal hyperostosis: clinical features and pathogenic mechanisms. Nat Rev Rheumatol 2013;9:741-50.

8 Kuperus JS, Waalwijk JF, Regan EA, et al. Simultaneous occurrence of ankylosing spondylitis and diffuse idiopathic skeletal hyperostosis: a systematic review. Rheumatology 2018;57:2120-8.

9 Slonimsky E, Leibushor N, Aharoni D, et al. Pelvic enthesopathy on $\mathrm{CT}$ is significantly more prevalent in patients with diffuse idiopathic skeletal hyperostosis (DISH) compared with matched control patients. Clin Rheumatol 2016;35:1823-7.

10 Haller J, Resnick D, Miller CW, et al. Diffuse idiopathic skeletal hyperostosis: diagnostic significance of radiographic abnormalities of the pelvis. Radiology 1989;172:835-9.

11 Leibushor N, Slonimsky E, Aharoni D, et al. CT abnormalities in the Sacroiliac joints of patients with diffuse idiopathic skeletal hyperostosis. AJR Am J Roentgenol 2017;208:834-7.

12 Latourte A, Charlon S, Etcheto A, et al. Imaging findings suggestive of axial spondyloarthritis in diffuse idiopathic skeletal hyperostosis. Arthritis Care Res 2018;70:145-52.

13 Teunissen FR, Verbeek BM, Cha TD, et al. Spinal cord injury after traumatic spine fracture in patients with ankylosing spinal disorders. J Neurosurg Spine 2017;27:709-16.

14 Westerveld LA, van Bemmel JC, Dhert WJA, et al. Clinical outcome after traumatic spinal fractures in patients with ankylosing spinal disorders compared with control patients. Spine J 2014;14:729-40.

15 Okada E, Tsuji T, Shimizu K, et al. CT-based morphological analysis of spinal fractures in patients with diffuse idiopathic skeletal hyperostosis. J Orthop Sci 2017;22:3-9.

16 Reinhold M, Knop C, Kneitz C, et al. Spine fractures in ankylosing diseases: recommendations of the spine section of the German Society for orthopaedics and trauma (DGOU). Global Spine $J$ 2018;8:56S-68. 
Correction: Imaging of diffuse idiopathic skeletal hyperostosis (DISH)

Mader R, Baraliakos X, Eshed I, et al. Imaging of diffuse idiopathic skeletal hyperostosis (DISH). RMD Open 2020;6:e01151.

The authors would like to alert the readers of the following.

The co-author Jorrit-Jan Verlaan's name was displayed incorrectly in the published version; this has since been amended. In addition, the co-author Fabiola Atzeni's affiliation has been updated to 'Rheumatology Unit, Department of Internal and Experimental Medicine, University of Messina, Messina, Italy'.

Open access This is an open access article distributed in accordance with the Creative Commons Attribution Non Commercial (CC BY-NC 4.0) license, which permits others to distribute, remix, adapt, build upon this work noncommercially, and license their derivative works on different terms, provided the original work is properly cited, appropriate credit is given, any changes made indicated, and the use is non-commercial. See: http://creativecommons. org/licenses/by-nc/4.0/.

(C) Author(s) (or their employer(s)) 2020. Re-use permitted under CC BY-NC. No commercial re-use. See rights and permissions. Published by BMJ.

RMD Open 2020;6:e001151corr1. doi:10.1136/rmdopen-2019-001151corr1

D Check for updates 